\title{
Preparación de morteros y hormigones no tradicionales a base de cemento portland, metacaolín y yeso $(15,05 \%)$
}

\author{
Manufacturing of mortars and concretes non-traditionals, by \\ portland cement, metakaoline and gypsum (15.05\%)
}

\author{
R.TALERO*,M.R.BOLLATI y F.HERNÁNDEZ** \\ * Instituto de Ciencias de la Construcción EduardoTorroja-CSIC \\ *^ Dpto. de Construc. y Técn. Arquitectónicas-E. T.S. de Arquitectura de Madrid-U. P.M.
}

Fecha de recepción: $25-\mathrm{XI}-97$

Fecha de aceptación: 8-IV-99

ESPAÑA

\section{RESUMEN}

En una exhaustiva investigación anterior(I), se pudo comprobar que la creación, evolución y desarrollo de los valores de resistencias mecánicas a compresión,RMC, $y$ flexotracción, $R M F$, proporcionados por probetas de $1 \times 1 \times 6 \mathrm{~cm}$, de mortero $1: 2,75$, selenitoso tipo ASTM C 452-68(2) -que habian sido preparadas con arena de Ottawa, cemento portland, $P-1\left(14,11 \% C_{3} A\right)$ o $P Y$ $6\left(0,00 \% \mathrm{C}_{3} \mathrm{~A}\right)$, metacaolin y yeso $\left(\mathrm{CaSO}_{4} 2 \mathrm{H}_{2} \mathrm{O}\right)$-, fue semejante a la que, comúnmente, desarrollan los morteros $y$ hormigones tradicionales de cemento portland. En el presente trabajo se exponen los resultados experimentales obtenidos de morteros y hormigones no tradicionales, preparados con dichos cementos ternarios, $C T$, siendo las proporciones porcentuales en masa ensayadas, cemento portland/metacaolín, las siguientes:80/20,70/30 y 60/40. Finalmente, el comportamiento de estos cementos frente el ataque del yeso, se ha determinado mediante los siguientes parámetros: incremento de longitud( $\triangle L \%)$, resistencias mecánicas a compresión, RMC, y flexotracción, $R M F, y$ energía de ultrasonido, EU.

Los resultados experimentales obtenidos con tales morteros y hormigones no tradicionales, han dado un aumento de los valores de $\triangle L, R M$ y EU, con la cantidad de metacaolín añadida.

\begin{abstract}
In a thorough previous research (1), it appeared that creation, evolution and development of the values of compressive mechanical strength(CS) and flexural strength(FS), measured in specimens $1 \times 1 \times 6 \mathrm{~cm}$ of mortar type ASTM C 452-68(2), manufactured by ordinary portland cement $P-1\left(14.11 \% C_{f} A\right)$ or $P Y-6\left(0.00 \% C_{f} A\right)$, metakaolin and gypsum $\left(\mathrm{CaSO}_{4} \cdot 2 \mathrm{H}_{2} \mathrm{O}\right)$-or ternary cements, $C T-$, were similar to the ones commonly developed in mortars and concretes of OPC. This paper sets up the experimental results obtained from non-traditional mortars and concretes prepared with such ternary cements -TC-, being the portland cement/metakaolin mass ratio, as follows: 80/20, 70/30 and 60/40. Finally, the behaviour of these cements against gypsum attack, has been also determined, using the following parameters: increase in iength( $\triangle L \%)$, compressive, $C S$, and flexural, FS, strengths, and ultrasound energy,UE.
\end{abstract}

Experimental results obtained from these non-traditional mortars and concretes, show an increase in length $(\Delta L)$, in $C S$ and FS, and in UE values, when there is addition of metakaolin. 


\section{INTRODUCCIÓN}

Ante todo, se desea puntualizar que este trabajo constituye una parte de una investigación más amplia (1), que, entre otros objetivos, pretendió obtener la caracterización sulfática de adiciones puzolánicas naturales y artificiales (y, posteriormente, siderúrgicas), mediante el método acelerado de ensayo ASTM C 452-68 (2). Y para alcanzar dicho objetivo (1) (20), una de las adiciones puzolánicas que se tuvo que seleccionar (3), fue metacaolín.

El metacaolín es una puzolana artificial de la que han existido -y existen- muchas citas bibliográficas, dado el enorme interés industrial que siempre ha despertado como material de construcción. De entre todas ellas -y por su relación con este trabajo- no deseamos dejar de citar aquí algunas(5) (6) (7) (8) y (9), ni tampoco los notables comentarios(10) (11) y réplica correspondiente (12) que suscitaron.Existen muchas más, si bien, con diferentes objetivos.

En este artículo, se exponen los resultados experimentales obtenidos con dicho metacaolín, al mezclarlo con los cementos portland P-1 y PY-6, respectivamente, en las proporciones porcentuales en masa siguientes: P-1/M y PY-6/M 80\%/20\%, 70\%/ $30 \%$ y $60 \% / 40 \%$, para ser ensayados mediante el citado método ASTM C 452-68 (2), sus consecuencias en morteros y hormigones y el análisis, estudio y discusión de los mismos.

\section{OBJETIVOS}

1. Obtener la caracterización sulfática del metacaolín utilizado, es decir, determinar su "carácter" o intencionalidad de comportamiento ante el ataque del yeso, cuando es añadido a un cemento portland, ya sea éste o no, de elevada resistencia a dicho ataque agresivo. En definitiva, determinar si se trata o no, de una puzolana "silícica" o "alumínica" o"sílicoaluminosa"o "alumino-silícica" o "sílico-férrica", etc. (1).

2. En función del "carácter" resultante anterior, preparar cementos, morteros y hormigones no tradicionales, con el metacaolín utilizado.

\section{PARTE EXPERIMENTAL}

Primeramente se prepararon los cementos binarios P-1/M y PY-6/M, 80/20,70/30 y 60/40 (relación en masa, cemento portland/metacaolín M). En la Tabla 1 , se pueden ver los resultados del análisis químico de ambos cementos portland, del metacaolín y del yeso utilizado como agresivo.

\section{INTRODUCTION}

First, it has to be specified that this paper represents a small part of a more extensive research (1) which was aimed, among other goals, at obtaining sulphate characterization of natural and artificial pozzolanic additions (and later, blast furnace slag), by accelerated testing method ASTM C 452-68 (2). To reach this goal (1) (20), one of the pozzolanic additions selected (3) was metakaolin.

Metakaolin is an artificial pozzolan which, since it has been and keeps being the object of a huge interest as construction material, has produced abundant bibliographical quotations. Among them those connected with this paper will be cited (5) (6) (7) (8) and (9), and some of the noteworthy comments (10) (11) and the corresponding answers (12) they arose, will be cited too. However, many more exist but they aim to different objectives.

This paper presents the experimental results obtained from mixing metakaolin with portland cements $P-1$ and $P Y-6$, respectively, mass ratio $80 \% / 20 \%, 70 \% / 30 \%$ and $60 \% / 40 \%$, tested by ASTM $C$ 452-68 method (2). The impact on mortars and concretes was determined through their analysis, study and discussion.

\section{OBJECTIVES}

1. To obtain the sulphatic characterization of the metakaolin, and thus, to determine its "behaviour" against gypsum attack, when added to a portland cement either of high or low sulfate resistance; and of course, to determine if metakaolin is "silicious" or "aluminous", or "silicic-aluminous", or "aluminoussilicic", or "silicic-ferrous", etc.(1), pozzolan.

2. To prepare non-traditional mortars and concretes with this metakaolin, in accordance with the resulting "behaviour".

\section{EXPERIMENTAL PROCESS}

First, binary cements $P-1 / M$ and $P Y-6 / M, 80 / 20$, $70 / 30,60 / 40$ (mass ratio portland cement/metakaolin M) were prepared. In Table 1, the results of the chemical analysis of both portland cements, of the metakaolin and the gypsum used as aggressive, are shown. 
TABLA $1 /$ TABLE 1

Análisis químico de los cementos portland, el metacaolín y el yeso.

Chemical analysis of portland cements, metakaoline and gypsum.

\begin{tabular}{|c|c|c|c|c|}
\hline \multirow{2}{*}{$\begin{array}{l}\text { DETERMINACIONES (\%) } \\
\text { DETERMINATIONS(\%) }\end{array}$} & \multicolumn{2}{|c|}{$\begin{array}{l}\text { CEMENTOS PORTLAND } \\
\text { PORTLAND CEMENTS }\end{array}$} & \multirow{2}{*}{$\begin{array}{c}\text { PUZOLANA } \\
\text { POZZOLAN } \\
\mathbf{M} \\
(2,55)^{*}\end{array}$} & \multirow[b]{2}{*}{$\begin{array}{l}\text { YESO } \\
\text { GYPSUM }\end{array}$} \\
\hline & $\begin{array}{c}\text { P-1 } \\
(3,08)^{*}\end{array}$ & $\begin{array}{l}\text { PY-6 } \\
(3,21)^{\star}\end{array}$ & & \\
\hline PF & 1,60 & 1,11 & 0,60 & - \\
\hline RI & 0,70 & 0,15 & 0,22 & 0,26 \\
\hline $\mathrm{SiO}_{2}$ & 19,18 & 21,70 & 73,53 & 0,04 \\
\hline $\mathrm{Al}_{2} \mathrm{O}_{3}$ & 6,44 & 1,52 & 23,11 & - \\
\hline $\mathrm{Fe}_{2} \mathrm{O}_{3}$ & 1,75 & 4,11 & 1,19 & - \\
\hline $\mathrm{CaO}$ & 63,94 & 67,97 & 0,63 & 32,54 \\
\hline MgO & 1,48 & 0,42 & 0,03 & 0,36 \\
\hline $\mathrm{SO}_{3}$ & 3,50 & 2,34 & - & 45,87 \\
\hline $\mathrm{Na}_{2} \mathrm{O}$ & 0,90 & 0.43 & 0,07 & 0,02 \\
\hline $\mathrm{K}_{2} \mathrm{O}$ & 0,52 & 0,20 & 0,70 & 0,01 \\
\hline $\mathrm{H}_{2} \mathrm{O}\left(40-217^{\circ} \mathrm{C}\right)$ & - & - & - & 20,13 \\
\hline $\mathrm{CO}_{2}\left(217-1000^{\circ} \mathrm{C}\right)$ & - & - & - & 0,75 \\
\hline Total & 100,01 & 99,95 & 100,05 & 99,98 \\
\hline $\mathrm{H}_{2} \mathrm{O}\left(105^{\circ} \mathrm{C}\right)$ & 0,24 & 0,22 & 0.16 & - \\
\hline $\mathrm{H}_{2} \mathrm{O}\left(40^{\circ} \mathrm{C}\right)$ & - & - & - & 0,41 \\
\hline $\mathrm{CaO}$ (libre) & 1,90 & 1,75 & - & - \\
\hline Total & 100,01 & 99,95 & 100,05 & 99,98 \\
\hline $\begin{array}{l}\text { SUP. ESPECIFICA BLAINE }\left(\mathrm{cm}^{2} / \mathrm{g}\right) \\
\text { SPF. SURF. }\left(\mathrm{cm}^{2} / \mathrm{g}\right)\end{array}$ & 3192 & 3287 & 3981 & - \\
\hline \multicolumn{5}{|c|}{ COMPOSICIÓN MINERALÓGICA (Bogue)(\%)/MINERALOGICAL COMPOSITION(\%) } \\
\hline $\mathrm{C}_{3} \mathrm{~S}$ & 51,05 & 79,43 & - & - \\
\hline $\mathrm{C}_{2} \mathrm{~S}$ & 16,48 & 2,29 & - & - \\
\hline$C_{3} A$ & 14,11 & 0,00 & - & - \\
\hline C4AF & 5,33 & 10,19 & - & - \\
\hline Cuarzo & - & - & $\cong 50$ & - \\
\hline $\mathrm{CaSO}_{4} .2 \mathrm{H}_{2} \mathrm{O}$ & - & - & - & 95,58 \\
\hline $\mathrm{CaSO}_{4} \cdot 1 / 2 \mathrm{H}_{2} \mathrm{O}+\mathrm{CaSO}_{4}$ & - & - & - & 2,47 \\
\hline $\mathrm{CaCO}_{3}$ & - & - & - & 0,75 \\
\hline $\mathrm{MgCO}_{3}$ & - & - & - & 0,81 \\
\hline \multicolumn{5}{|c|}{ - Peso especifico $\left(\mathrm{g} / \mathrm{cm}^{3}\right) /$ Specific weight $\left(\mathrm{g} / \mathrm{cm}^{3}\right)$} \\
\hline
\end{tabular}

El metacaolín, cuyo contenido de cuarzo era de, aproximadamente el $50 \%$, se preparó calcinando caolín a $800^{\circ} \mathrm{C}$ durante el tiempo necesario. A continuación se verificó su carácter puzolánico mediante el ensayo de Frattini (4),véase la Figura 1.

A continuación, cada cemento portland y cada cemento binario anterior, se ensayó según el método acelerado de ensayo ASTM C 452-68 (2). Los parámetros determinados en cada familia de probetas de mortero $1: 2,75$, selenitoso (cemento más yeso $=7,0 \% \mathrm{SO}_{3}$ ) fueron:incremento de longitud, $\Delta \mathrm{L}(\%)$, resistencias mecánicas a compresión, RMC, y flexotracción, RMF, y contenido de sulfatos, $\mathrm{SO}_{4}{ }_{4}$, en las aguas de conservación de las probetas tipo ASTM C 452-68. Este último

\section{FIGURA 1/FIGURE 1}

Ensayo de Frattini. Edad: 7 días

Frattini test. Age: 7 days

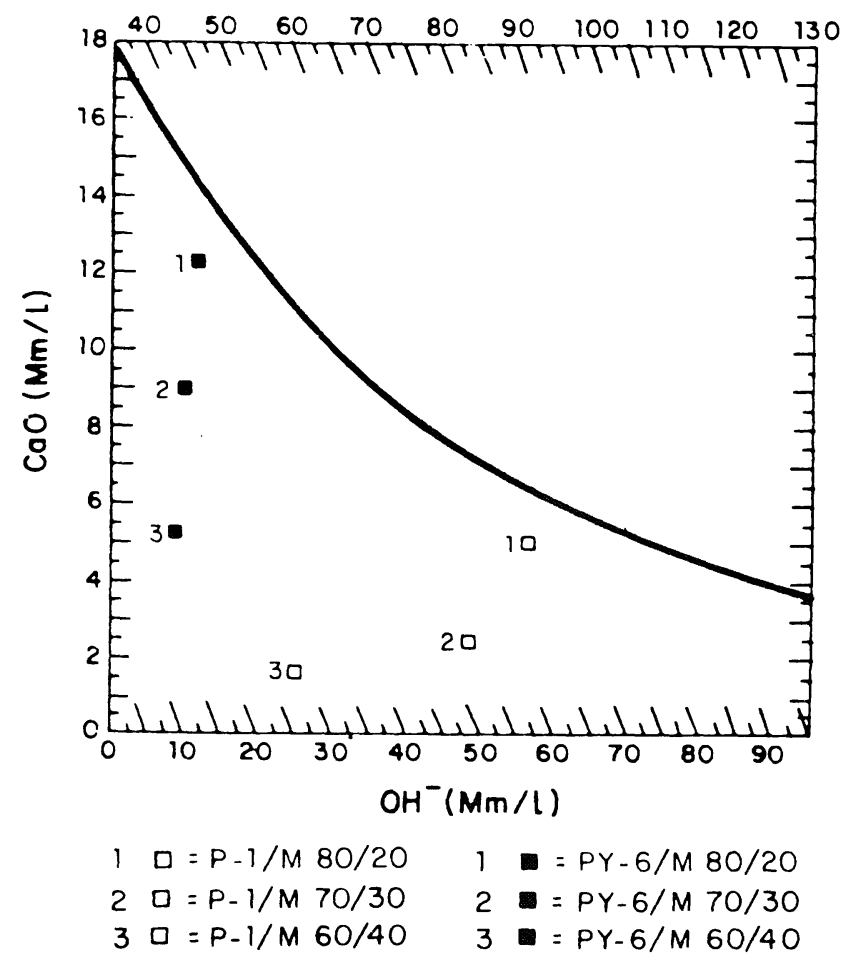

The metakaolin, whose quartz content was $50 \%$, was prepared by burning kaolin at $800{ }^{\circ} \mathrm{C}$ for the time required. Following, its pozzolanic character was verified by Frattini test (4), see Figure 1.

Later, each portland cement and each binary cement was tested according to the ASTM C 452-68 test (2). Parameters determined were the following: increase in length $\Delta L(\%)$, compressive strength -CS-, flexural strength $-\mathrm{FS}$ - and sulphate content- $\mathrm{SO}_{4}{ }^{=}$-in storage water where ASTM 452-68 type specimens were kept. This last parameter was determined by 
parámetro se determinó periódicamente en las aguas de conservación de las probetas, precipitando, en medio ácido clorhídrico, los sulfatos, en forma de sulfato de bario.

Una vez obtenidos y analizados los resultados experimentales obtenidos con los morteros, se prepararon una serie de hormigones que ratificaron el mejor comportamiento de los cementos ternarios,CT, preparados ante el ataque del yeso, si la cantidad añadida de éste, era adecuada. La dosificación de éstos hormigones fue la siguiente: (A) Hormigones cuyo CT tenía $7,0 \% \mathrm{SO}_{3}(=15,05 \%$ de yeso), y (B) Hormigones cuyo CT tenía $21,0 \% \mathrm{SO}_{3}(45,16 \%$ de yeso), véase la Tabla 2 .

Con estas dosificaciones se prepararon probetas de hormigón de tres tipos:

1. Prismáticas, de $10 \times 10 \times 50 \mathrm{~cm}$, en las que se determinó su $\Delta \mathrm{L}(\%)$, en función del tiempo.

2. Cilíndricas, de $\phi 15 \times 18 \mathrm{~cm}$, en las que se determinó su RMC $\left(\mathrm{kp} / \mathrm{cm}^{2}\right)$ y resistencia mecánica a tracción indirecta o ensayo "brasileño", BS $\left(\mathrm{kp} / \mathrm{cm}^{2}\right)$, a las edades de 7, 28 y 90 días. periodically testing the storage water, where sulphates precipitate in the chloride acid environment as barium sulphate.

Once experimental results obtained from mortars were analysed, a series of concretes were prepared, which ratify the better behaviour of the terciary cements (TC) prepared when addition of gypsum was adequate. The dosage of these concretes was : (A) TC with $7.0 \% \mathrm{SO}_{3}(=15.05 \%$ of gypsum), and (B) TC with $21.0 \% \mathrm{SO}_{3}(=45.16 \%$ of gypsum), see Table 2.

With these dosages, concrete specimens of three types, were prepared:

1. Prisms of $10 \times 10 \times 50 \mathrm{~cm}$ where the $\Delta L(\%)$ was determined as a function of time,

2. Cylinders of $\phi 15 x 18 \mathrm{~cm}$, where the $C S\left(\mathrm{kp} / \mathrm{cm}^{2}\right)$ and compressive strength to indirect traction ("brasilian" test) $B S\left(\mathrm{kp} / \mathrm{cm}^{2}\right)$, were determined at age 7,28 and 90 days,

TABLA 2/TABLE 2

Dosificación de los hormigones $\left(\mathrm{kp} / \mathrm{m}^{3}\right) /$ Concretes dosages $\left(\mathrm{kp} / \mathrm{m}^{3}\right)$

\begin{tabular}{|c|c|c|c|c|c|c|c|c|c|}
\hline \multirow{3}{*}{$\begin{array}{l}\text { PROPORCIÓN } \\
\text { MEZCLAS } \\
\text { MIXTURE } \\
\text { PROPORTIONS } \\
\text { IDENTIFICACIONN } \\
\text { IDENTIFICATION }\end{array}$} & \multicolumn{9}{|c|}{ YESO/GYPSUM } \\
\hline & \multicolumn{3}{|c|}{$\underset{\text { WITH }}{\mathrm{CON}} 7 \% \mathrm{SO}_{3}$} & \multicolumn{3}{|c|}{$\begin{array}{l}\mathrm{CON}_{21 \% \mathrm{SO}_{3}} \\
\text { WITH }^{21 \%}\end{array}$} & \multicolumn{3}{|c|}{$\begin{array}{l}\text { SN YESO } \\
\text { WITHOUT GYPSUM }\end{array}$} \\
\hline & C & $\mathbf{Y}$ & AC & C & $\mathbf{Y}$ & AC & C & $\mathbf{Y}$ & AVC \\
\hline P1 & 282,0 & 39,0 & 0,500 & 193,0 & 159,5 & 158,5 & 315 & - & 158,5 \\
\hline PY6 & 280,0 & 41,0 & 0,500 & 174,5 & 193,0 & 158,5 & 315 & - & 158,5 \\
\hline P1/M 80/20 & 276,5 & 44,5 & 0,525 & 190,0 & 164,0 & 170,0 & 315 & - & 158,5 \\
\hline P1/M 70/30 & 274,5 & 46,5 & 0,585 & 188,0 & 166,0 & 189,0 & 315 & - & 158,5 \\
\hline P1/M 60/40 & 273,0 & 48,0 & 0,665 & 186,0 & 168,0 & 189,0 & 315 & - & 158,5 \\
\hline PY6R 80/20 & 276,0 & 45,0 & 0,525 & 185,0 & 170.0 & 189,0 & 315 & - & 158,5 \\
\hline PY6/M 70/30 & 247.5 & 46,5 & 0,585 & 184,0 & 171,0 & 189,0 & 315 & - & 158,5 \\
\hline PY6/M 60/40 & 273,0 & 48,0 & 0,665 & 183,0 & 172,0 & 189,0 & 315 & - & 158,5 \\
\hline \multicolumn{10}{|c|}{ 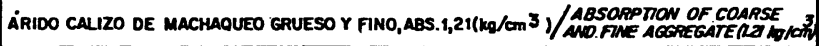 } \\
\hline $10-20 \mathrm{~mm}$ & \multicolumn{3}{|c|}{643,0} & \multicolumn{3}{|c|}{654,0} & \multicolumn{3}{|c|}{654,0} \\
\hline 5-10 mm & \multicolumn{3}{|c|}{436.0} & \multicolumn{3}{|c|}{436,0} & \multicolumn{3}{|c|}{436,0} \\
\hline Fino/Fine & \multicolumn{3}{|c|}{1012,0} & \multicolumn{3}{|c|}{1017,0} & \multicolumn{3}{|c|}{1017,0} \\
\hline \multicolumn{10}{|c|}{ PROPIEDADES DEL HORMIGÓN FRESCO/FRESH CONCRETE PPOPERTES } \\
\hline $\begin{array}{l}\text { P.ESPECIFTCO } \\
\text { APARENTE } \\
\text { UNT WE/GHT }\end{array}$ & \multicolumn{3}{|c|}{$2410 \mathrm{~kg} / \mathrm{m}^{3}$} & \multicolumn{3}{|c|}{$2250 \mathrm{~kg} / \mathrm{m}^{3}$} & \multicolumn{3}{|c|}{$2250 \mathrm{~kg} / \mathrm{m}^{3}$} \\
\hline $\begin{array}{l}\text { ASENTAMIENTO } \\
\text { SLUMP }\end{array}$ & \multicolumn{3}{|c|}{$0 \mathrm{~cm}$} & \multicolumn{3}{|c|}{$6 \mathrm{~cm}$} & \multicolumn{3}{|c|}{$6 \mathrm{~cm}$} \\
\hline $\begin{array}{l}\text { COMPACTACÓN } \\
\text { COMPACTAMON }\end{array}$ & \multicolumn{3}{|c|}{$\begin{array}{l}\text { VIBRADO } \\
\text { VIBRATED }\end{array}$} & \multicolumn{3}{|c|}{$\begin{array}{l}\text { PICADO } \\
\text { RODDED }\end{array}$} & \multicolumn{3}{|c|}{$\begin{array}{l}\text { PICADO } \\
\text { RODOED }\end{array}$} \\
\hline $\begin{array}{l}\text { ProbeTAS } \\
\text { SPECMUES }\end{array}$ & \multicolumn{3}{|c|}{$10 \times 10 \times 50$ y $15 \times 18$} & \multicolumn{3}{|c|}{$7,5 \times 15$} & \multicolumn{3}{|c|}{$7,5 \times 15$} \\
\hline
\end{tabular}


3. Cilíndricas, de $\phi 7,5 \times 15 \mathrm{~cm}$, en las que se determinó su Acción Sinérgica Expansiva, ASE.

Todas las probetas se fabricaron con un sistema de vibrocompactación de amplitud y frecuencia variables que permite obtener las probetas en el laboratorio, en las mismas condiciones que en obra.

Las probetas prismáticas y las cilíndricas de $\phi 15 \times 18 \mathrm{~cm}$ y $\phi 7,5 \times 15 \mathrm{~cm}$, se conservaron bajo agua potable filtrada, hasta que fue constante su $\Delta \mathrm{L}(\%)$ para las primeras. Se obtuvieron sus valores de RMC y de resistencia mecánica a tracción indirecta o ensayo "brasileño", BS, de las segundas, y se degradaron las terceras.

Una probeta cilíndrica, de $\phi 15 \times 18 \mathrm{~cm}$ fue regada intermitentemente con agua, de modo semejante al caso anterior, mientras que era sometida al ensayo de energía de ultrasonido, EU.

\section{RESULTADOS EXPERIMENTALES OBTENIDOS}

- Del ensayo de Frattini (4): Figura 1.

- Del método acelerado de ensayo ASTM C 452-68

(2): Figuras $2(\Delta \mathrm{L}) ; 3(\mathrm{Vcl}=[\Delta \mathrm{L}(\%) /$ día]; $4(\mathrm{RMC} \mathrm{y}$

$\mathrm{RMF})$ y $5\left(\mathrm{SO}_{4}{ }^{\circ}\right)$.

- Del ensayo de los hormigones: $\Delta \mathrm{L}(\mathrm{mm} / \mathrm{m})$, Figura 5 ; $\mathrm{RMC}\left(\mathrm{kp} / \mathrm{cm}^{2}\right)$ y BS $\left(\mathrm{kp} / \mathrm{cm}^{2}\right)$, Tabla 3; EU (24), Figura 7.

- Del agua de consistencia normal, tiempo de fraguado y estabilidad de volumen (14) (15), de los CT: Tabla 4.

\section{DISCUSIÓN E INTERPRETACIÓN}

De los resultados experimentales obtenidos se pudo deducir que el $7,0 \% \mathrm{SO}_{3}(=15,05 \%$ de yeso $)$ del método ASTM C 452-68 (2), había actuado lógicamente como agresivo en el caso de los cementos portland P-1 y PY-6 cuando se ensayaron solos, y, en especial, con el P-1, pero no así, en cambio, cuando estuvo presente la puzolana $\mathrm{M}$ en los cementos ternarios, CT, en cuyo caso, actuó como regulador de fraguado.

Para confirmar esta deducción, a los CT P-1/M y PY-6/M 80/20,70/30 y 60/40, se les determinó, además, sus Tiempos de Fraguado (Principio y Fin) y Estabilidad de Volumen(14) (15), respectivamente. Los valores obtenidos fueron los que aparecen en la Tabla 4. Como se puede apreciar en dicha tabla, todos
3.Cylinders of $\phi 7.5 \times 15 \mathrm{~cm}$, where Expansive Synergic Action, ESA, was determined.

All specimens were made through vibro-compatation system of variable amplitude and frecuency which allows to manufacture similar specimens in laboratory as in work site.

Prismatic and cylindrical specimens of $\phi 15 \times 18 \mathrm{~cm}$ and $\phi 7.5 \times 15 \mathrm{~cm}$, were soaked in filtered potable water until the $\Delta L(\%)$ was constant, in the first set. $C S$ values and indirect traction values ("brasilian specimens" BS) were obtained in the second set, and the third set was degradated.

One cylindrical specimen of $\phi 15 x 18$ was watered intermittently with similar water to the one used in the test before, while being submitted to an ultrasound energy test (UE).

\section{EXPERIMENTAL RESULTS}

\author{
- Frattini test (4): Figure 1. \\ - ASTM C 452-68 (2): Figures 2( $\triangle L)$; \\ $3(\mathrm{Vcl}=[\Delta L(\%) /$ day $]), 4(\mathrm{CS}$ and $\mathrm{FS})$ and $5\left(\mathrm{SO}_{4}{ }^{\circ}\right)$
}

- Concretes test: $\Delta L(\mathrm{~mm} / \mathrm{m})$, Figure 5; CS and FS $\left(\mathrm{kp} / \mathrm{cm}^{2}\right)$ and BS (kp/cm $\left.{ }^{2}\right)$, Table 3; and UE (24), Figure 7.

- Water of normal consistency, setting time and volume stability (14) (15), of TC: Table 4.

\section{DISCUSSION AND INTERPRETATION}

From experimental results it was inferred that 7,0\% $\mathrm{SO}_{3}$ (=15.05\% of gypsum) of ASTM C 452-68 (2) test, had acted, as expected, as aggressive in the case of portland cements $P-1$ and $P Y-6$ when they were plain cements and particularly in the case of P-1; but it acted differently when the pozzolan $M$ was present since this one acted as setting regulator.

To confirm what had been inferred, Setting Time (Start and End) and Volume Stability (14) (15) were determined. Values obtained are shown in Table 4. 


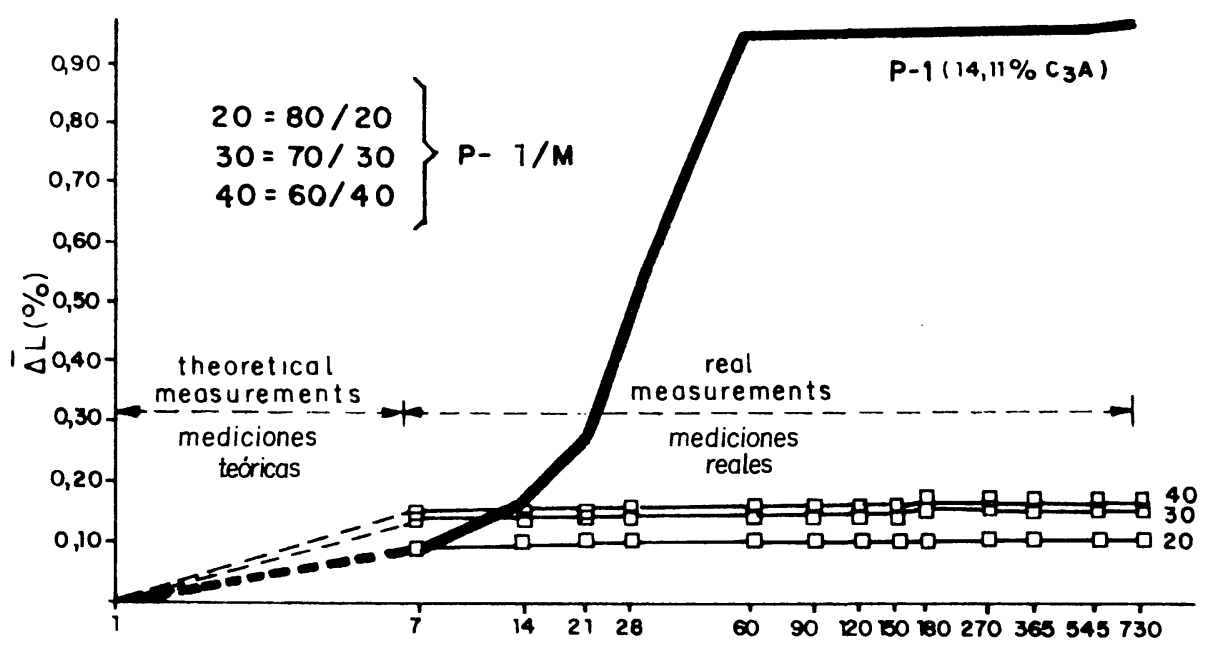

$\operatorname{EDAD}($ días) $/ A G E$ (days)

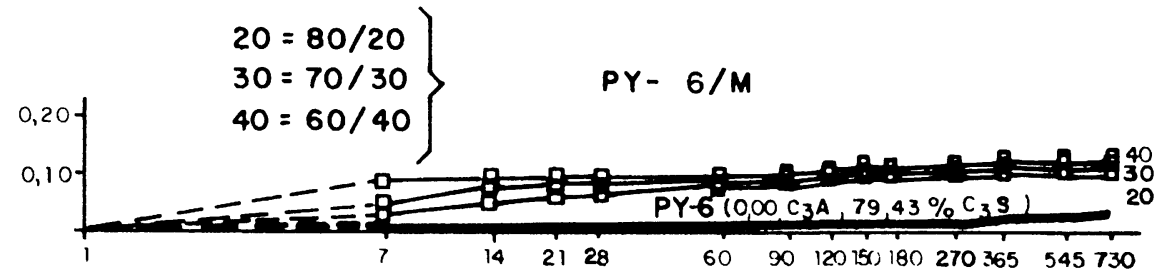

$\operatorname{EDAD}($ días)/AGE (days)

Figura 2.- Probetas: 1"x1"x11 1/4"; Parámetros: Incremento de Longitud (\%); Morteros: ASTM C 452 - 68 (2); Cementos: OPC $=$ P-1, SRPC $=$ PY -6; Puzolana: $M$

Figure 2.- Specimens: 1"xl"x111/4"; Parameter: Increase in Length (\%); Mortars: ASTM C 452 - 68 (2); Cements: $O P C=P-1, S R P C=P Y-6 ;$ Pozzolan: $M$

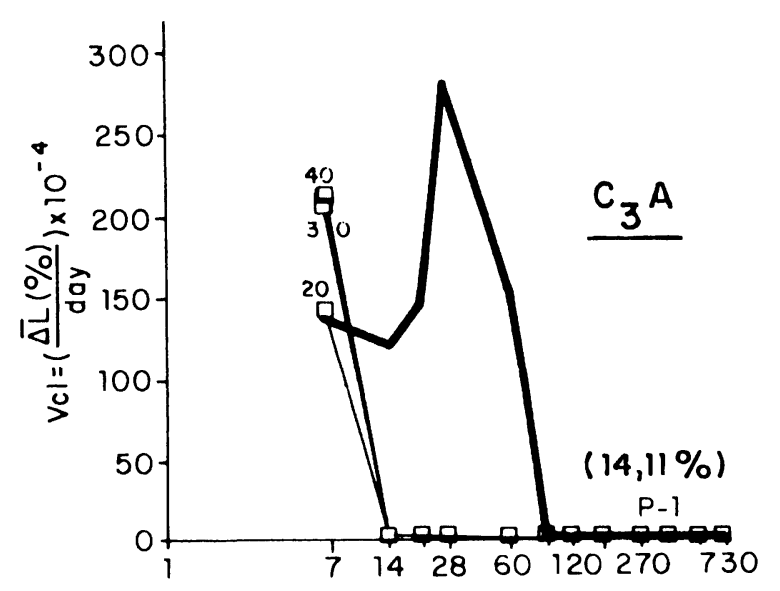

$\operatorname{EDAD}($ dias) $/ A G E$ (days)

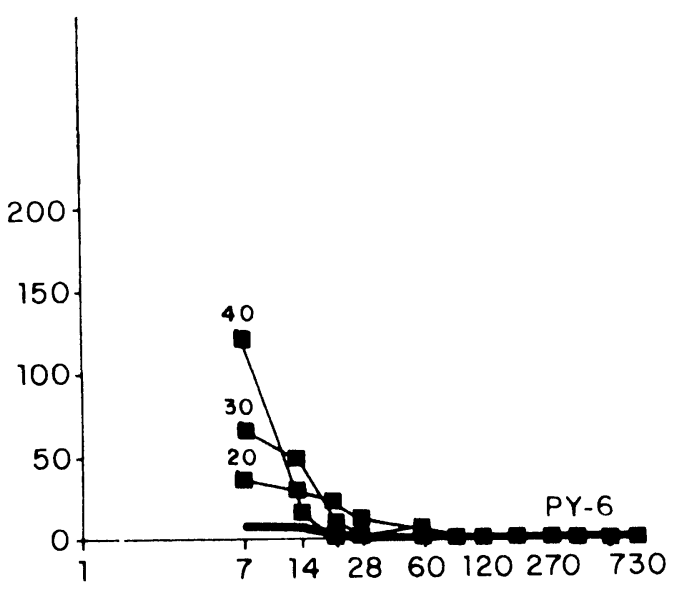

$\operatorname{EDAD}($ dias) $/ A G E$ (days)

Figura 3.- Probetas: 1"xl"x11 1/4"; Parámetros: Vcl = $\Delta \mathrm{L}$ (\%)/día; Morteros: ASTM C 452 - 68 (2); Cementos: OPC = P-1, SRPC = PY-6; Puzolana: M

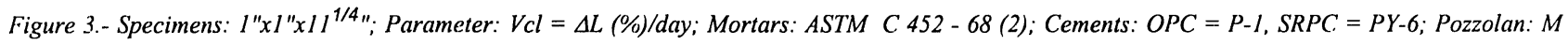



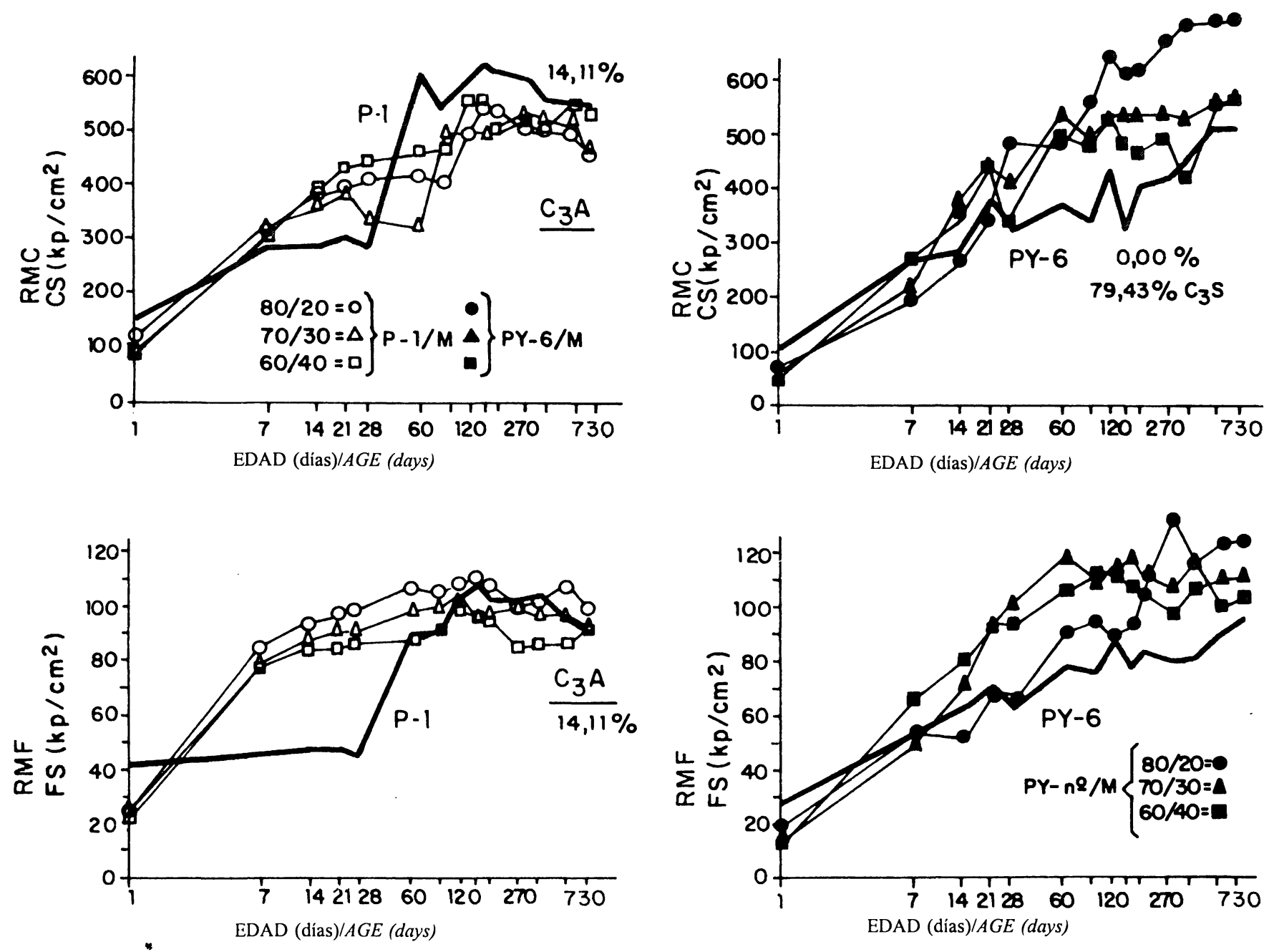

Figura 4.- Probetas: $1 \times 1 \times 6 \mathrm{~cm}$; Parámetros: Resistencias Mecánicas a Flexotracción, FS, y Compresión, CS; Morteros: ASTM C 452 - 68 (2); Cementos: $\mathrm{OPC}=\mathrm{P}-1, \mathrm{SRPC}=\mathrm{PY}-6 ;$ Puzolana: $\mathrm{M}$

Figure 4.- Specimens: $1 \times 1 \times 6 \mathrm{~cm} ;$ Parameter: Flexural Strength, FS, and Compressive Strength, CS; Mortars: ASTM C $452-68$ (2); Cements: OPC = P-1, SRPC $=$ PY-6; Pozzolan: $M$
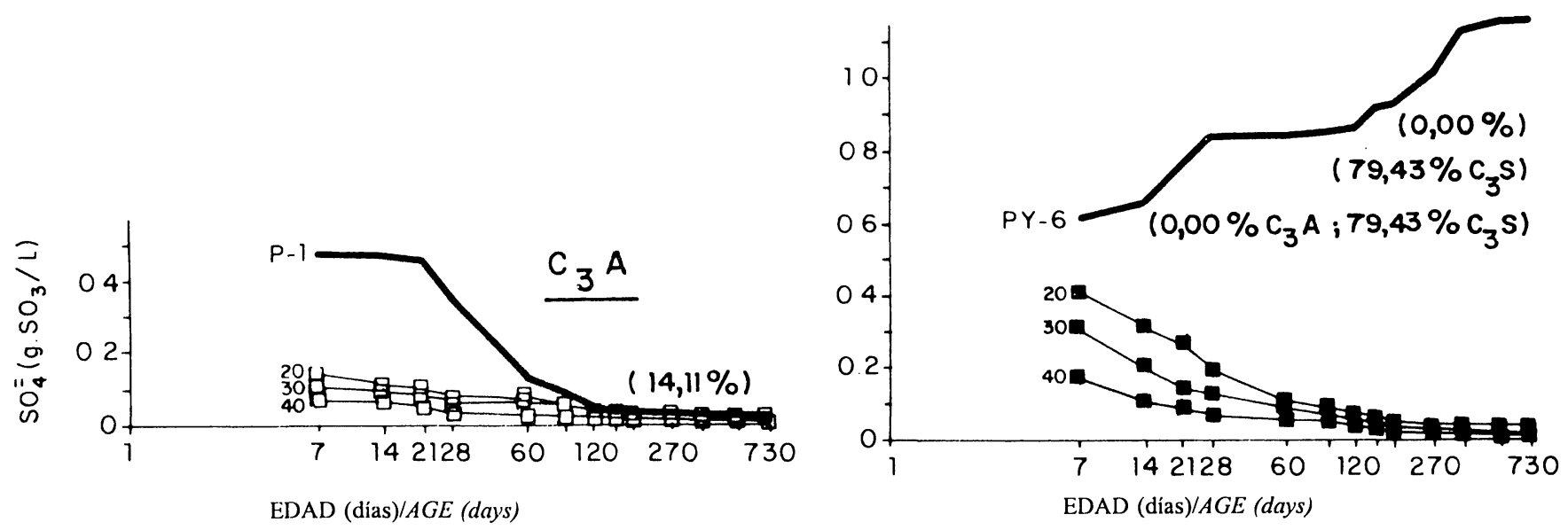

Figura 5.- Probetas: $1 \times 1 \times 6 \mathrm{~cm}$; Parámetros: Contenido de $\mathrm{SO}_{4}=$ en las aguas de conservación a diferentes edades; Morteros: ASTM C 452 - 68 (2); Cementos: $\mathrm{OPC}=\mathrm{P}-1, \mathrm{SRPC}=\mathrm{PY}-6$; Puzolana: $\mathrm{M}$

Figure 5.- Specimens: $1 \times 1 \times 6 \mathrm{~cm} ;$ Parameter: Conservation water $\mathrm{SO}_{4}{ }^{=}$contents at different ages; Mortars: $A S T M$ C $452-68$ (2); Cements: OPC $=$ $P-1, S R P C=P Y-6 ;$ Pozzolan: $M$ 


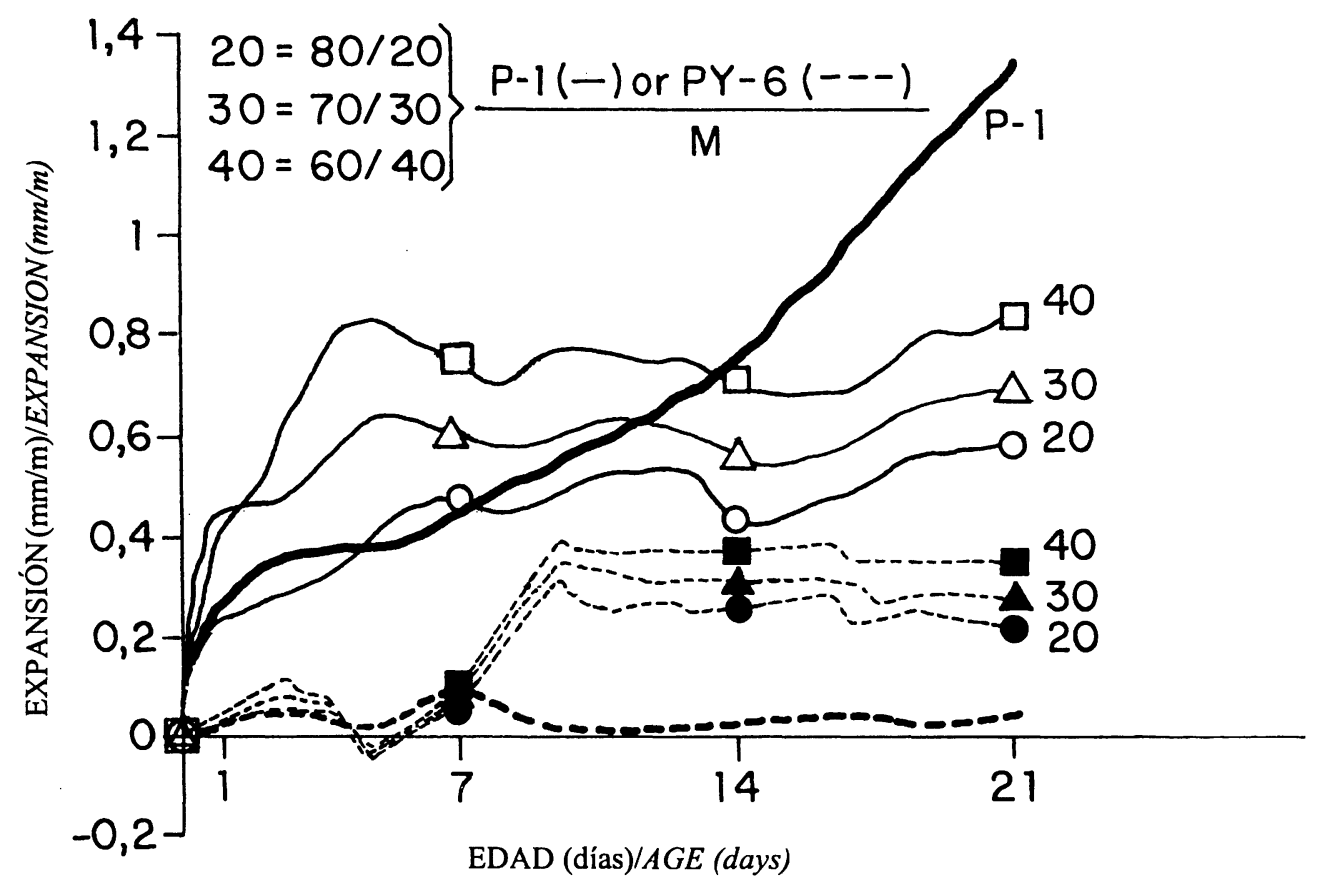

Figura 6.- Probetas: 10x10x50 cm; Hormigones: Dosificación (ver Tabla 2); Cementos con yeso (7,0\% $\left.\mathrm{SO}_{3}\right)$ : OPC $=\mathrm{P}-1, \mathrm{SRPC}=\mathrm{PY}-6$ y $6 \mathrm{CT}$ : P-1/M $80 / 20$

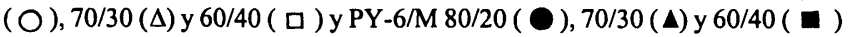

Figure 6.- Specimens: 10x10x50 cm; Concretes: Dosage (see Table 2); Cements with gypsum $\left(7.0 \% \mathrm{SO}_{3}\right): \mathrm{OPC}=P-1, S R P C=P Y-6$ and 6 CT: $P-1 / M$

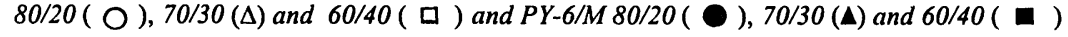

\section{TABLA3/TABLE 3}

Resistencias mecánicas de los hormigones $\left(\mathrm{kp} / \mathrm{cm}^{2}\right)$

Mechanical strengths of the different concretes $\left(\mathrm{kp} / \mathrm{cm}^{2}\right)$

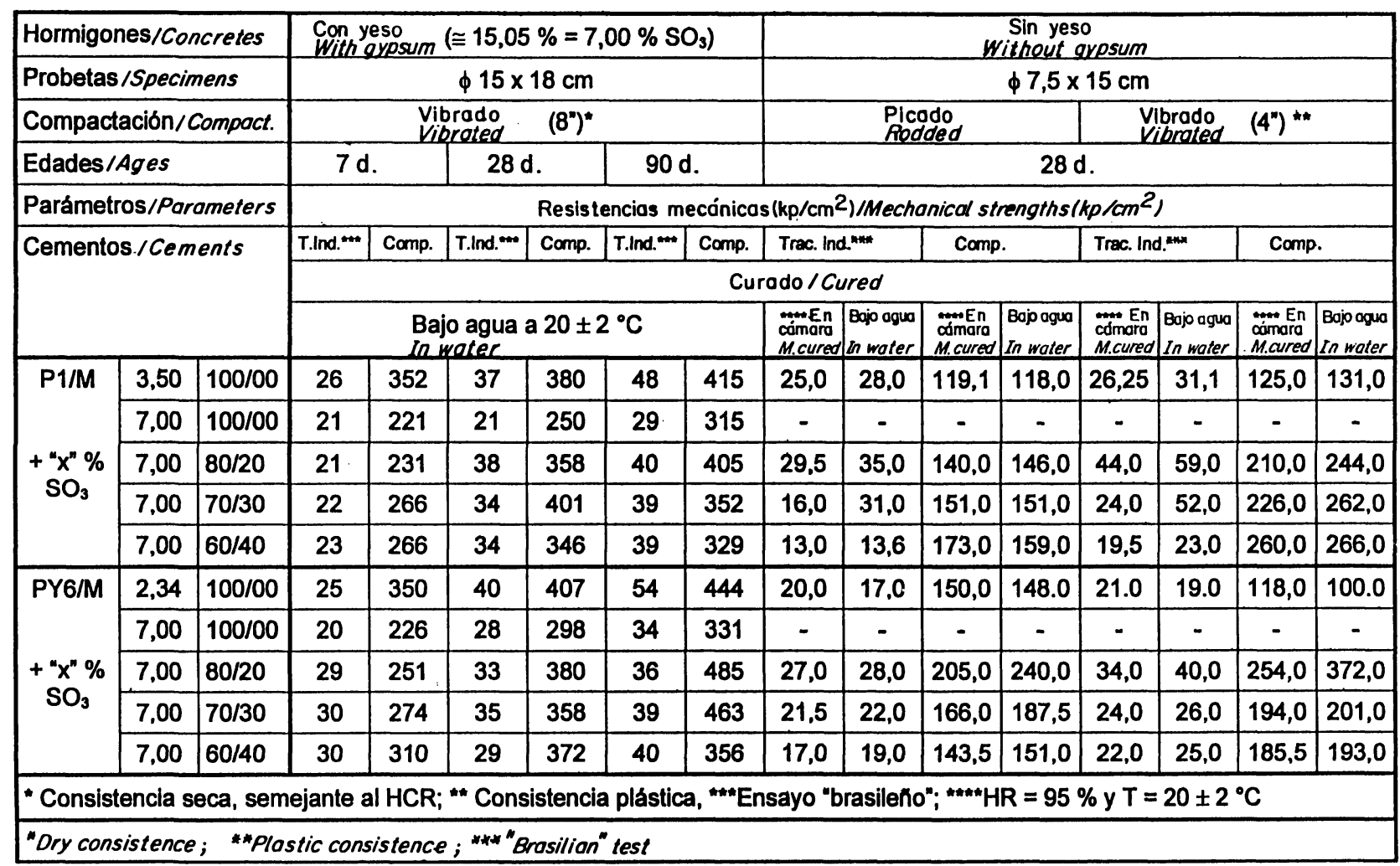




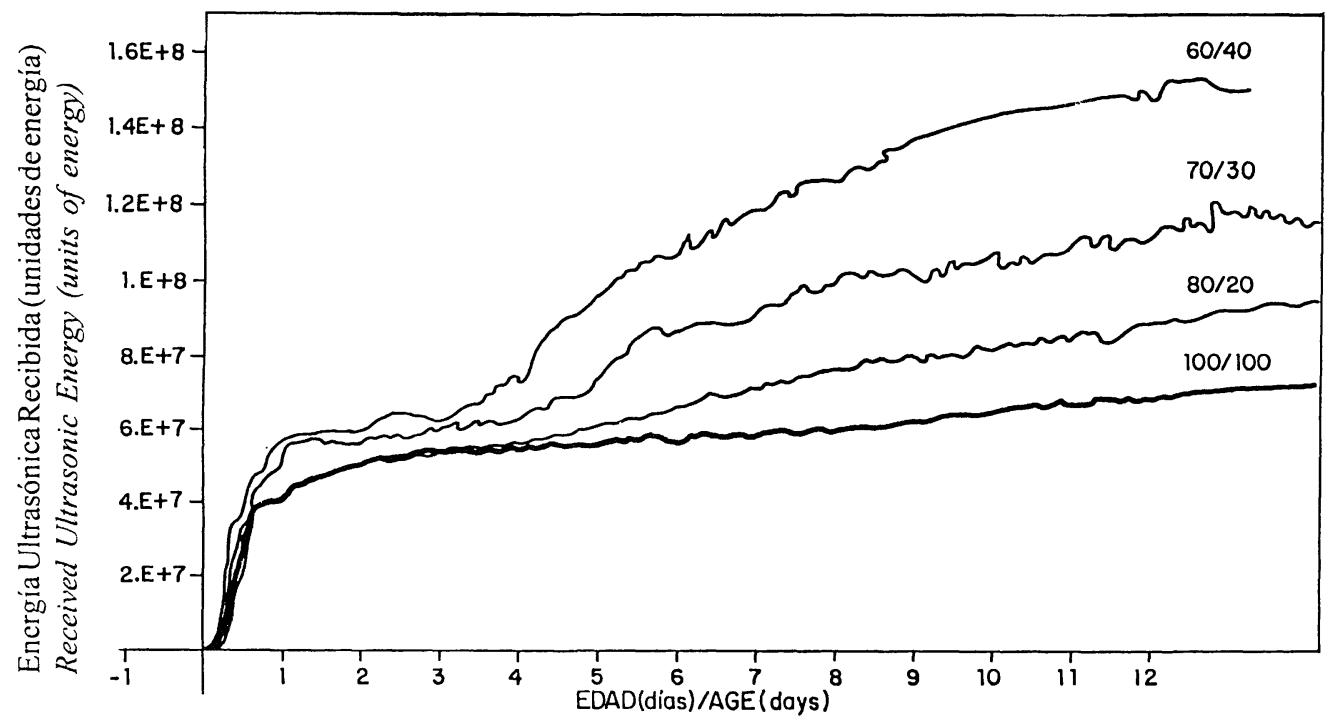

Figura 7.- Probetas: $\phi 15 \times 18 \mathrm{~cm}$; Parámetro: EU; Hormigones: Dosificación (ver Tabla 2); Cementos con yeso $\left(7,() \% \mathrm{SO}_{3}\right): \mathrm{OPC}=\mathrm{P}-1(-)$ y $3 \mathrm{CT}=\mathrm{P}-1 / \mathrm{M} 80 / 20(\mathrm{O}), 70 / 30(\Delta)$ y 60/40 ( $\left.\mathrm{C}\right) ;$ Cámara Húmeda: $21 \pm 2{ }^{\circ} \mathrm{C}$ y $95 \%$ HR.

Figure 7.- Specimens: $\phi 15 \times 18 \mathrm{~cm}$; Parameter: UE; Concretes: Dosage (see Table 2); Cements with gypsum $\left(7.0 \% \mathrm{SO}_{3}\right): O P C=P-1(-)$ and $3 \mathrm{CT}=P_{-} 1 / \mathrm{M} \mathrm{80/20}(\mathrm{O}), 70 / 30(\Delta)$ and 60/40 ( $\square$ ); Moist Closet: 21 $\pm 2{ }^{\circ} \mathrm{C}$ and $95 \% \mathrm{RH}$

\section{TABLA 4/TABLE 4}

Tiempo de fraguado $(\mathrm{h}, \mathrm{m})$

Time of setting $(h, m)$

\begin{tabular}{|c|c|c|c|c|c|c|c|c|c|c|c|c|c|c|}
\hline \multirow{3}{*}{\multicolumn{3}{|c|}{$\begin{array}{l}\text { CEMENTOS } \\
\text { CEMENTS }\end{array}$}} & \multirow{4}{*}{\begin{tabular}{|c|}
$\begin{array}{c}\text { Pasta de } \\
\text { consistencia } \\
\text { normal } \\
N . \text { consisteny } \\
(\%)\end{array}$ \\
26.0 \\
\end{tabular}} & \multirow{2}{*}{\multicolumn{3}{|c|}{$\begin{array}{l}\text { Tiempo de fraguado } \\
\text { (aguja de Vicat) } \\
\text { Time of set ting (Vicat test) }\end{array}$}} & \multicolumn{8}{|c|}{ Agujas de Le Chatelier $(\mathrm{mm}) /$ Le Chatelier Needles $(\mathrm{mm})$} \\
\hline & & & & & & & \multirow{2}{*}{$\frac{100^{\circ} \mathrm{C}}{7^{\circ}}$} & \multicolumn{7}{|c|}{$21 \pm 2^{\circ} \mathrm{C}$} \\
\hline & & & & Inicial & Final & TF. & & $7^{*}$ & $12^{*}$ & $21^{*}$ & $28^{n}$ & $60^{\circ}$ & $90^{*}$ & $120^{*}$ \\
\hline \multirow{4}{*}{$\begin{array}{l}\mathrm{P} 1 / \mathrm{M}+ \\
{ }^{*} \mathrm{x}^{*} \% \\
\mathrm{SO}_{3}\end{array}$} & 3,50 & $100 / 00$ & & $2 \mathrm{~h} \mathrm{00m}$ & $2 \mathrm{~h} 39 \mathrm{~m}$ & Oh $39 \mathrm{~m}$ & 0,70 & 1,70 & 1,70 & 1,70 & 1,70 & 1,70 & 1,70 & 1,70 \\
\hline & 7,00 & $80 / 20$ & 29.6 & $3 \mathrm{~h} 15 \mathrm{~m}$ & 4h $10 \mathrm{~m}$ & Oh $55 \mathrm{~m}$ & 0,50 & 3,33 & 5,65 & 7,20 & 9,35 & 12,00 & 12,15 & 12,15 \\
\hline & 7,00 & $70 / 30$ & 30,8 & $3 \mathrm{~h} 05 \mathrm{~m}$ & ath $10 \mathrm{~m}$ & 1h 05m & 0,50 & 5,15 & 6,00 & 6,33 & 6,65 & 6,71 & 6,75 & 7,75 \\
\hline & 7,00 & $60 / 40$ & 32,8 & $3 \mathrm{~h} 15 \mathrm{~m}$ & 4h $10 \mathrm{~m}$ & Oh $55 \mathrm{~m}$ & 0,50 & 5,75 & 5,75 & 5,75 & 5,75 & 5,75 & 5,75 & 5,75 \\
\hline \multirow{4}{*}{$\begin{array}{l}\mathrm{PY} 6 / \mathrm{M} \\
+\mathrm{x}^{\prime \prime} \% \\
\mathrm{SO}_{3}\end{array}$} & 2,34 & $100 / 00$ & 21,2 & Oh $05 \mathrm{~m}$ & Oh $25 \mathrm{~m}$ & Oh $20 \mathrm{~m}$ & 1.00 & 0,30 & 0,30 & 0,30 & 0,30 & 0.30 & 0,30 & 0,30 \\
\hline & 7,00 & $80 / 20$ & 27,2 & $6 \mathrm{~h} 15 \mathrm{~m}$ & $8 \mathrm{~h} 10 \mathrm{~m}$ & in $55 \mathrm{~m}$ & 0,50 & 2,00 & 3,50 & 3,55 & 3,60 & 3,90 & 4,00 & 4,50 \\
\hline & 7,00 & $70 / 30$ & 28,8 & $5 \mathrm{~h} 45 \mathrm{~m}$ & $6 \mathrm{~h} 55 \mathrm{~m}$ & th $10 \mathrm{~m}$ & 0,00 & 3,20 & 3,70 & 3,80 & 4,00 & 4,60 & 4,60 & 4,70 \\
\hline & 7,00 & $60 / 40$ & 31,6 & $5 \mathrm{~h} 50 \mathrm{~m}$ & $7 \mathrm{~h} 35 \mathrm{~m}$ & 1h $45 \mathrm{~m}$ & 0,50 & 5,00 & 5,00 & 5,00 & 5,00 & 5,00 & 5,00 & 5,00 \\
\hline
\end{tabular}

los valores se encuentran dentro de lo especificado al respecto por las normas ASTM C 191-82 (14), EN 196-3 (15) y ASTM C 845-80 (16), menos las probetas de los CT P-1/M 70/30 y 60/40, que proporcionaron unos valores de $\Delta \mathrm{L}_{7 \mathrm{~d}}$ del $0,141 \% \mathrm{y}$ $0,148 \%$, respectivamente, es decir, $>0,10 \%$ (16). Por
This Table shows that all values are within AST'M stantards C 191-82 (14), EN 196-3 (15) and ASTM C 845-80 (16), unless specimens of TC P-1/M 70/30 and $60 / 40$ which gave values of $\Delta L_{7 d}$ of $0.141 \%$ and $0.148 \%$, respectively, which means $>0.10 \%(16)$; reason why those two TCs cannot be considered 
esta razón, ambos CT no pudieron ser considerados expansivos (16), el resto, en cambio, si. A pesar de lo cual, en todos ellos, la cantidad de yeso aportada como agresivo $\left(=15,05 \%=7,0 \% \mathrm{SO}_{3}\right)$, no actuó como tal agresivo, sino como regulador de fraguado. Por esta razón todos estos cementos ternarios,CT, están de acuerdo con la propuesta de Matousek y Sauman (5).

En definitiva, cuando el contenido de yeso de los CT $(15,05 \%)$ fue el adecuado, la acción del mismo sobre el metacaolín no pudo calificarse de nociva, siendo, por tanto, intrascendente, que no equivalente, el tipo de cemento portland que lo acompañaba.

Por el contrario, cuando dicha cantidad de yeso se triplicó, entonces resultó muy nociva y, por tanto, inadecuada. De aquí que las probetas de hormigón picado de $\phi 7,5 \times 15 \mathrm{~cm}$, de los CT P-1/M y PY-6/M, se hubieran auto-destruído por ataque del yeso, 1,5 años antes (Fotos 1-6).

Y ambos comportamientos diferentes del metacaolín ante el ataque del yeso, positivo en el primer caso y negativo en el segundo, han sido debidos a la Accíon Sinérgica Expansiva, ASE (1), que se ha originado a tiempo y destiempo, respectivamente, entre: la ettringita de rápida formación (25) o de origen alúmina reactiva, $\mathrm{Al}_{2} \mathrm{O}_{3}{ }^{\mathrm{r}}$, o alúmina tetra o penta-coordinada (26), de la puzolana $M$, en este caso, y la ettringita de lenta formación (25) o de origen $\mathrm{C}_{3} \mathrm{~A}$ del cemento portland -el P-1 principalmente, en este caso-, que le acompañaba (1) (21).

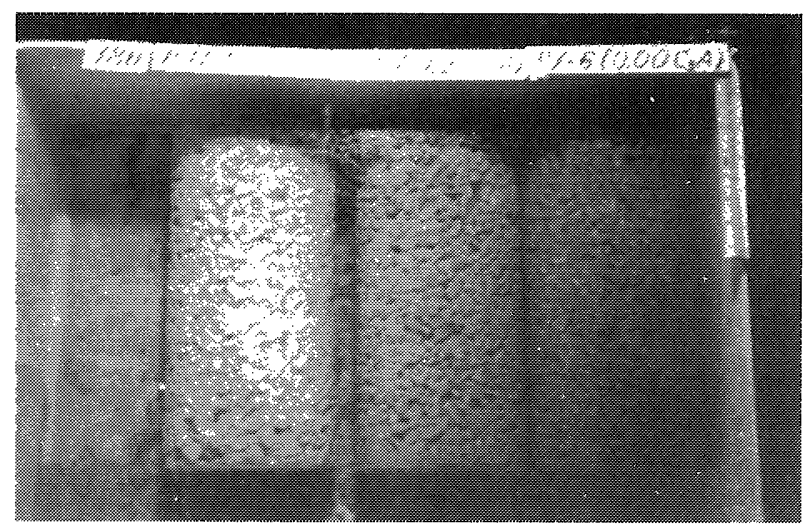

Foto 1.- Probetas de $\phi 7,5 \times 15 \mathrm{~cm}$ de hormigón picado y de árido calizo, do los cementos portland, CP, P $1\left(14,11 \% \mathrm{C}_{3} \mathrm{~A}\right)$ y $\mathrm{PY}-6\left(0,00 \% \mathrm{C}_{3} \mathrm{~A}\right)$. El contenido inicial de yeso de ambos CP fue del $45,16 \%$. Edad: 180 días. Lil CP P. $31\left(7,63 \% \mathrm{C}_{3} \mathrm{~A}\right)$, no se ha incluido en esta investigación.

Photo 1." Specimens $(\phi 7.5 \times 15 \mathrm{~cm})$ of rodded concrete and limestone aggregates that were manufactured with the $O P C, P-1\left(14,11 \% C_{3} A\right)$ and $P Y-6\left(0.00 \% C_{3} A\right)$. Initial gypsum content for both $O P C 45,16 \%$ Age: 180 days. The OPC P-3I was not take into account for this research. expansive (16), while the rest already is. However, in all of them, the amount of the gypsum added as aggressive $\left(=15.05 \%=7.0 \% \mathrm{SO}_{3}\right)$ did not acted as aggressive but as setting regulator or setting control, reason why all these CT are in agreement with Matousek and Sauman's proposal (5).

In short, when gypsum content in TC was appropriate $(15.05 \%)$, its effect on metakaolin could not be considered harmful, being indifferent the kind of portland cement used.

On the other hand, when the gypsum amount was tripled, it appeared to be very noxious, therefore inadequate, which explains why specimens of rodded concrete of $\phi 7.5 \times 15 \mathrm{~cm}$ of the TC P-1/M and PY-6/M were self-destroyed after gypsum attack one year and a half, earlier. Photos 1-6.

Both metakaolin behaviours were different when facing the gypsum attack: positive in the first case, negative in the second, due to the Synergic Expansive Action, SEA (1) which appeared in-time and off-time, respectively, between: quick formation ettringite (25), or of reactive alumina origin $\mathrm{Al}_{2} \mathrm{O}_{3}$, or tetra, or penta-coodinated alumina (26), of pozzolan $M$ in this case, and slow formation ettringite (25) or from the $C_{3} A$ of portland cement, mainly $P-1$ in this case, which was involved in it (1) (21).

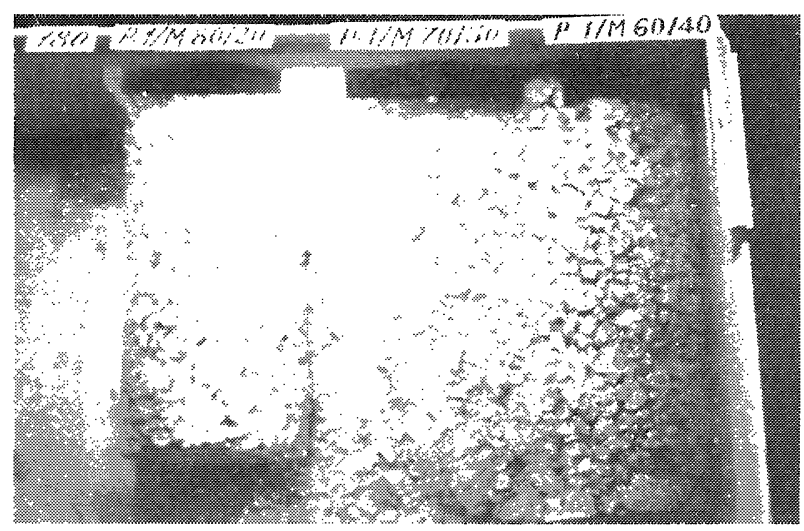

Foto 2.- Probetas de $\$ 7,5 \times 15 \mathrm{~cm}$ de hormigón picado y de árido calizo, de la familia de los cementos ternarios, CT, P.1/M. El contenido inicial de yeso de ambos CT fue del 45,16\%. Ldad: 180 dias. Obsérvese que las probetas $70 / 30$ y $60 / 40$, ya han sido destruidas por ataque selenitoso.

Photo 2.- Specimens $(\phi 7.5 \times 15 \mathrm{~cm})$ of rodded concrete and limestone aggregates of the ternary cements families, $P_{-}-1 / M$. Initial gy:psum content for each ternary cement was 45.16\%. Age: 180 days. See that $70 / 30$ and 60/40 specimens, have been cracked due to gypsum attack. 


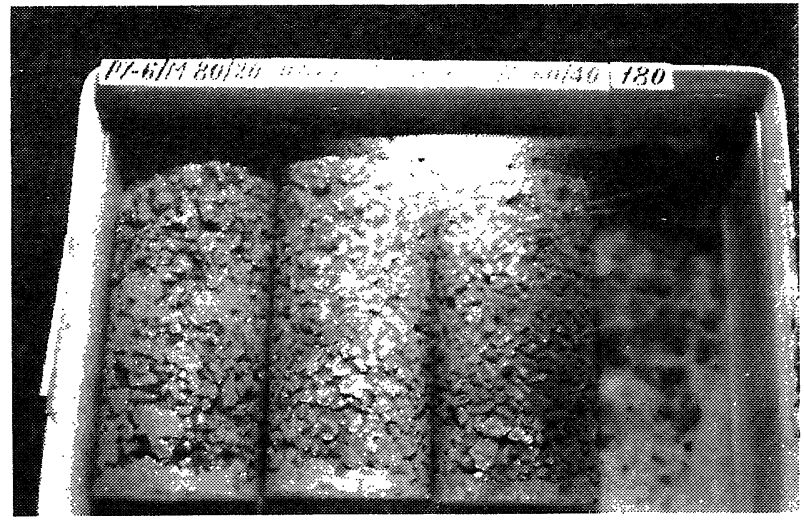

Foto 3.- Idem Foto 2, sólo que la familia de los cementos ternarios, PY-6/M, no ha comenzado a sufrir aún los efectos del ataque selenitoso. Edad: 180 días.

Photo 3.- Same as Photo 2, but PY-6/M specimens have not started to suffer the effects of gypsum attack. Age: 180 days.

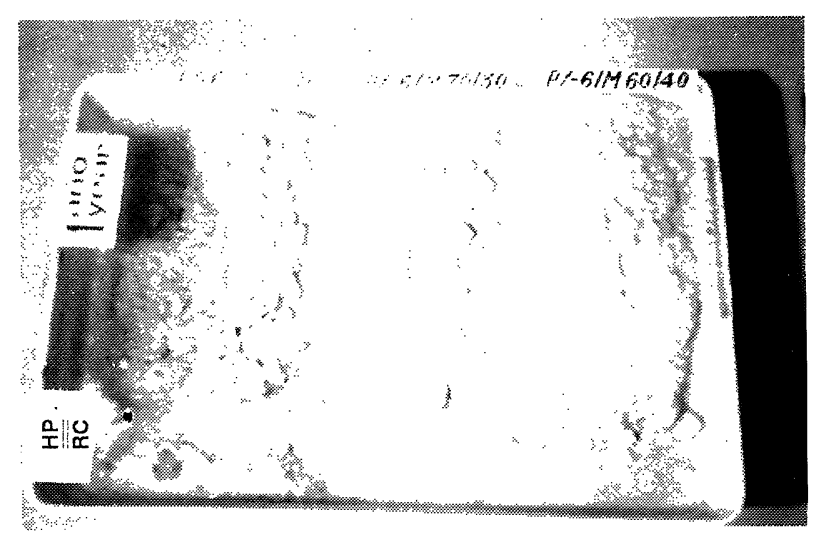

Foto 5.- Idem Foto 3, sólo que los malos efectos del ataque selenitoso, propiciado en esta ocasión, casi en exclusiva, por el metacaolín $\mathrm{M}$, han llegado a su fin. Edad: 1 año.

Photo 5.- Same as Photo 3, but all damage due to gypsum attack which in this case was mainly originated by $M$ metakaolin, have abready taken place. Age: 1 year.

Por último y en base a que la consustancial expansividad de la ettringita, de cualquier origen y etiología, lleva aparejada además, paralelamente y, en cualquier caso, con su formación, un proceso colmatador de huecos, se ha de destacar el buen comportamiento mostrado por los hormigones preparados con los CT que contenían $7,0 \% \mathrm{SO}_{3}$, en el ensayo de EU. Ya que, y por tal motivo, deberían de haber dado por resultado en dicho ensayo de EU, semejante proporcionalidad a la mostrada por el parámetro $\Delta \mathrm{L}$ con la adición de metacaolín, como así ha ocurrido en esta investigación.

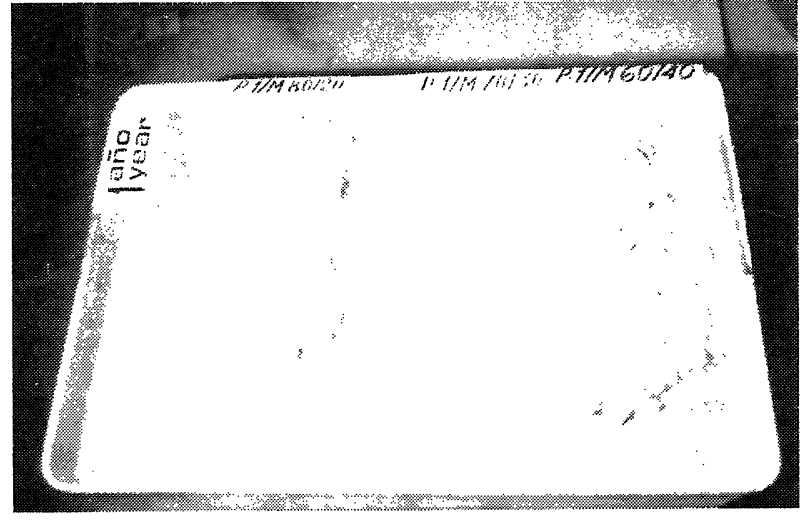

Foto 4.- Idem Foto 2, sólo que los malos efectos del ataque selenitoso han llegado a su fin. Edad: 1 año.

Photo 4.- Same as Photo 2, but all damage due to gypsum attack have abready taken place. Age: 180 days.

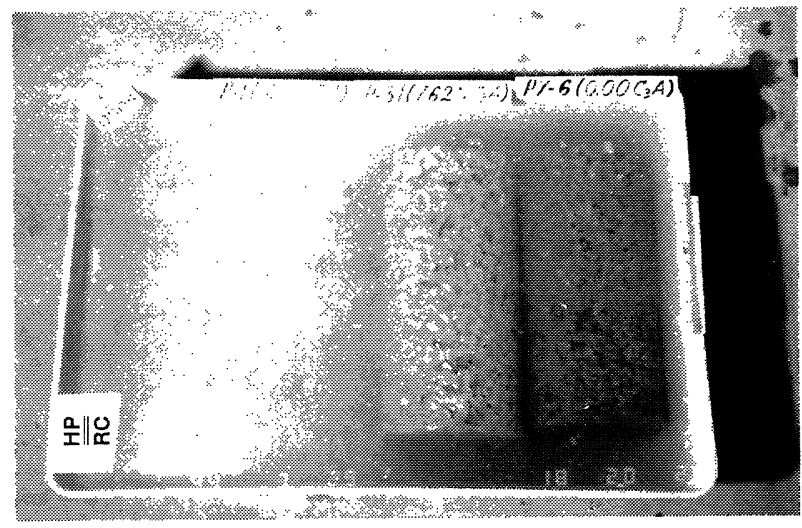

Foto 6.- Idem Foto 1, sólo que los malos efectos del ataque selenitoso, propiciado en esta ocasión, casi en exclusiva, por el cemento portland P-1 $\left(14,11 \% \mathrm{C}_{3} \mathrm{~A}\right)$, han llegado a su fin. Edad: 2 años.

Photo 6.- Same as Photo 1, but all damage due to gypsum attack which in this case was mainly originated by OPC $P-1\left(14.11 \% C_{3} A\right)$, have abready taken place. Age: 2 years.

Finally, on the basis that the inherent expansibility of ettringite -whatever its origin and etiology could be- bears in its formation a stuffing process of hollows, the attention must be drawn on the good behaviour shown by concretes prepared with TCs containing $7.0 \% \mathrm{SO}_{3}$, in UE test, since for that reason, they should have given as result, a similar proportionment to the one shown in parameter $\Delta L$, with addition of metakaolin, as happened all through this investigation. 


\section{CONCLUSIONES}

$1^{\text {a }}$. De acuerdo con los resultados experimentales obtenidos y la propuesta de R.Talero (1) (20), el metacaolín utilizado en este trabajo pudo ser calificado como puzolana "alumínica". Este tipo de puzolanas, ya fueren naturales o artificiales:

(a) Facilitan el ataque del yeso a los cementos portland con los que se mezclaren, acortando notablemente su durabilidad, aun cuando dichos cementos portland sean de elevada resistencia al citado medio agresivo y, sobre todo, si los mismos son utilizados y puestos en obra cual si de cementos portland puros se tratasen, $y$

(b) Dosificadas adecuadamente con yeso -mediante el método ASTM C 452-68, adaptado de acuerdo con la propuesta de R.Talero (1) (22) (determinación del "óptimo" de $\mathrm{SO}_{3}$ de un CT semejante)-, aun sin dosificar, elevan las resistencias mecánicas a compresión, principalmente, de los cementos portland $\mathrm{y}$, sobre todo, a edades iniciales, y más aún, si sus productos derivados, hormigones, morteros y pastas, se curan con agua el tiempo necesario y a ser posible, aun estando en su encofrado (1).

$2^{\mathrm{a}}$. El $15,05 \%$ de yeso que se le añadió a cada cemento portland con metacaolín ensayado según el método ASTM C 452-68, no actuó como agresivo, sino como "regulador de fraguado" (esta cantidad de yeso puede que sea menor que la "óptima" para dicho fin, determinăda mediante el citado método de ensayo ASTM C 452-68, adaptado por R.Talero (1) (22)).

- En cambio, cuando dicha cantidad de yeso se triplicó, entonces se comportó como agresivo.

- Por consiguiente, en el primer caso, la cantidad de yeso añadida $(15,05 \%)$, se pudo definir como adecuada, (que no "óptima"(23)). En el segundo $(45,16 \%)$, no.

$3^{\text {a }}$. Según la norma ASTM C 845-80, todos los CT ensayados en este trabajo podrían ser considerados, en principio, como cementos expansivos menos el P-1/M $70 / 30$ y $60 / 40$, puesto que sus valores de $\Delta \mathrm{L}_{7 \mathrm{~d}}(\%)$ respectivos,fueron menores que $0,10 \%$.

$4^{\mathrm{a}}$. Los cementos ternarios, $\mathrm{CT}$, ensayados en este trabajo podrían ser también cementos sin retracción si su cantidad óptima de $\mathrm{SO}_{3}$ hubiese sido $<7,0 \%$, pero determinada, en cualquier caso, para este otro fin, mediante el método ASTM C 452-68, adaptado por R.Talero (1) (22).

$5^{\text {a }}$. La energía de ultrasonido ha demostrado su utilidad para poder caracterizar este tipo de hormigones

\section{CONCLUSIONS}

$1^{a}$ In accordance with experimental results and R.Talero's proposal (1) (20), the metakaolin used in this work could be classfied as "aluminous" or "aluminic" pozzolan. This kind of pozzolans, either natural or artificial,

(a) Fake easier the gypsum attack in any portland cement blended with it, decreasing their durability even in those with high sulfate resistant, and, mainly, if utilized and treated as if they were plain portland cements;

(b) With the proper gypsum dosage -by ASTM C 452-68 and adapted according to Talero's proposal (1) (22) (determination of the "optimum" $\mathrm{SO}_{3}$ of a similar TC)-, and even without excess of gypsum, they mainly reinforce the CS of portland cements, specially at early ages; and still more, if derivative products -concretes, mortars and pastes- are cured for a long enough time, and if possible, still being inside the formwork (1).

$2^{a}$. The $15.05 \%$ gypsum added to each portland cement with metakaolin, tested according to ASTM C 452-68 method, did not acted as agressive but as "setting regulator" or "setting control" (this amount of gypsum could be considered scarcely lower than the "optimum" for such a goal), determined using the ASTM 452-68 method adapted by $R$. Talero (1) (22).

- However when this gypsum amount was tripled, it behaved as aggressive.

- Therefore, in the first case, the amount of gypsum added (15.05\%) is considered adequate (not "optimum"(23)). In the second case with addition of $45.16 \%$, it was not.

$3^{a}$. According to standard ASTM C 845-80, all TCs tested in this work could be considered expansive cements, unless $P-1 / M 70 / 30$, and $60 / 40$, since their respective $\Delta L_{7 d}(\%)$ was smaller than $0,10 \%$.

$4^{a}$. In this work TCs tested could have also been cements without retraction if their optimum amount of $\mathrm{SO}_{3}$ had been $<7,0 \%$, but determined in any case for this purpose, with method ASTM C 452-68 adapted by R.Talero (1) (22).

$5^{a}$. Ultrasound energy, UE, has revealed its usefulness in characterizing this kind of special 
especiales y la puzolana artificial que los constituye: metacaolín.

concretes and its constituent: this artifical pozzolan named metakaolin.

\section{BIBLIOGRAFÍA}

(1) R.TALERO: Doctorate Thesis,Complutense University of Madrid, Ftad.C. Químicas, 20 nov. 1986.

(2) ASTM C 452-68 Standard.

(3) W.EITEL: Journal:Am.Concr.Inst. 28(7), 679-98(1957).

(4) N.FRATTINI: Ann.Chim.Applicata, 39, 616-20(1949).

(5) M.MATUSEK and Z.SAUMAN: Cem.Concr.Res., Vol.4, No .1 ,pp. 113-22, january.

(6) A.ARAIBIA, M.CHABANET, J.L.BOSC et J.PERA: 2nd.CANMET/ACI International Conference on Durability of Concrete.Supplemetary Papers, pp.385-406, Montreal, Canada, 1991.

(7) M.MURAT: Cem.Concr.Res., Vol.4,Nº.4,pp.113(1974).

(8) M.MURAT and C.COMEL:Cem.Concr.Res., Vol.13, Nº.2, pp. 259-66, march.1983.

(9) M.MURAT: Cem.Concr.Res., Vol.13, №.4,pp. 511-18, sept.1983.

(10) P.K.MEHTA: Cem.Concr.Res., Vol.4, No.4, pp. 113 (1974).

(11) P.K.MEHTA: Cem.Concr.Res., Vol.4, №.4, pp. 683-84 (1974).

(12) S.CHATTERJI: Cem.Concr.Res., Vol.4, No.4.pp. 687-88 (1974).

(13) M.MATOUSEK and Z.SAUMAN: Cem.Concr.Res., Vol.4, №.4, pp. 687-88 (1974).

(14) Pliego de Recepción de Cementos RC-75(BOE núm. 206 de 28-08-75).

(15) ASTM C 191-82 Standard.

(16) EN 196-3 Standard = UNE 80-102-88 Standard: Time of Setting and Stability in Volume (Le Chatelier Neddles's method).

(17) ASTM C 845-80 Standard.

(18) ASTM C 187-86 Standard.

(19) ASTM C 563-84 Standard.

(20) R.TALERO: Journal of Materials in Civil Engineering, Vol.2, №.2, may 1990.

(21) R.TALERO: ACAA.10th. Intern.Coal Ash Symposium, Orlando-Florida-USA, january 1993.

(2) R.TALERO: Ciclo de Conferencias sobre "Las Puzolanas Naturales, Cenizas Volantes y Similares en la Construcción, Cemento y Hormigón". EOI de Madrid; 9-11 de febrero de 1988.

(23) R.TALERO: Ciclo de Conferencias sobre "Las Puzolanas naturales, Cenizas Volantes y similares en la Construcción, cemento y hormigón". EOI de Madrid; 9-11 de febrero de 1988.

(24) M-R.BOLLATI: 6th. Intern.Symp. on Concrete Roads, pp. 83-92, Madrid, 8-10 oct. 1990.

(25) R.TALERO:C.C.R.,Vol.26, Nº.8,pp.1277-1283, 1996.

(20) J.SANZ, A.MADANI, J.M.SERRATOSA, J.S.MOYA y S.AZA: J.Am.Ceram.Soc. 71, [10], C-418-C-421 (1988). 